\title{
Comprehension of Grammatical and Emotional Prosody Is Impaired in Alzheimer's Disease
}

\author{
Vanessa Taler \\ Bloomfield Centre for Research in Aging and Centre for \\ Research on Language, Mind and Brain, McGill University \\ Howard Chertkow \\ Bloomfield Centre for Research in Aging, Department of \\ Neurology and Neurosurgery, and Centre for Research on \\ Language, Mind and Brain, McGill University
}

\author{
Shari R. Baum \\ School of Communication Sciences \& Disorders and Centre for \\ Research on Language, Mind and Brain, McGill University \\ Daniel Saumier \\ Department of Neurology and Neurosurgery and Centre for \\ Research on Language, Mind and Brain, McGill University
}

\begin{abstract}
Previous research has demonstrated impairment in comprehension of emotional prosody in individuals diagnosed with Alzheimer's disease (AD). The present pilot study further explored the prosodic processing impairment in $\mathrm{AD}$, aiming to extend our knowledge to encompass both grammatical and emotional prosody processing. As expected, impairments were seen in emotional prosody. AD individuals were also found to be impaired in detecting sentence modality, suggesting that impairments in affective prosody processing in $\mathrm{AD}$ may be ascribed to a more general prosodic processing impairment, specifically in comprehending prosodic information signaled across the sentence level. AD participants were at a very mild stage of the disease, suggesting that prosody impairments occur early in the disease course.
\end{abstract}

Keywords: grammatical prosody, emotional prosody, Alzheimer's disease, comprehension

Alzheimer's disease (AD) is an irreversible, progressive neurodegenerative brain disorder. The earliest symptoms include short- and long-term memory problems, but typically encompass other cognitive functions. There is now strong evidence indicating that memory impairments in AD may co-occur with problems in language skills (e.g., Hodges \& Patterson, 1995; for a review, see Caramelli, Mansur, \& Nitrini, 1998). Such impairments may make it more difficult for the patient to communicate with others, leading to communication breakdown that often contributes to increasing caregiver burden and conflicts in social relationships. A deeper understanding of speech and language impairments in $\mathrm{AD}$ thus has the potential for benefit in a number of areas, including improved interpersonal

Vanessa Taler, Bloomfield Centre for Research in Aging and Centre for Research on Language, Mind and Brain, McGill University; Shari R. Baum, School of Communiction Sciences \& Disorders and Centre for Research on Language, Mind and Brain, McGill University; Howard Chertkow, Bloomfield Centre for Research in Aging, Department of Neurology and Neurosurgery, and Centre for Research on Language, Mind and Brain, McGill University; Daniel Saumier, Department of Neurology and Neurosurgery and Centre for Research on Language, Mind and Brain, McGill University.

The present research has been supported by a pilot project grant awarded to the second and fourth authors from the Centre for Research on Language, Mind and Brain, as well as CIHR grant (11290) to the second author. Vanessa Taler is supported by a postdoctoral fellowship from the Alzheimer's Society of Canada/Fonds de la Recherche en Santé du Québec. We are grateful to Isabelle Peretz for providing stimuli used in Task 1, and to Shelley Solomon, Chris Hossein and Renee Kaminski for assistance in patient recruitment.

Correspondence concerning this article should be addressed to Shari R. Baum, School of Communication Sciences \& Disorders, McGill University, 1266 Pine Avenue West, Montreal, QC H3G 1A8. E-mail: shari.baum@mcgill.ca interaction, reduction in personal, marital, and caregiver stress, and a better understanding of the factors underlying behavioral disturbances.

Among the language skills affected by AD are those related to understanding prosody, which includes the alterations in pitch, rhythm, and loudness that convey information additional to the linguistic content. Speech prosody provides various types of communicative information, from cues to a speaker's emotional intent to patterns of stress or emphasis within an utterance, to cues to syntactic structure and lexical stress patterns. All of these prosodic functions are signaled to varying degrees by the same three primary acoustic parameters: fundamental frequency (F0; associated with perceived pitch), duration (perceived as length), and amplitude (loudness). Despite being signaled by the same acoustic cues, both production and perception or comprehension of the different functions of prosody may be independently affected by neurological damage (see, e.g., Baum \& Pell, 1999).

With respect to $\mathrm{AD}$, the majority of studies on prosodic processing to date have focused on emotional or affective prosody. For example, a number of studies have demonstrated that individuals with $\mathrm{AD}$ manifest impairments in processing prosodic information relating to emotional tone (Albert, Cohen, \& Koff, 1991; Allender \& Kaszniak, 1989; Cadieux \& Greve, 1997; Cohen \& Brosgole, 1988; Roberts, Ingram, Lamar, \& Green, 1996). Such impairments are more severe and appear earlier than those affecting other linguistic capacities (Testa, Beatty, Gleason, Orbello, \& Ross, 2001; Kertesz, 1982), and have been shown to worsen as dementia becomes more severe (Roberts et al., 1996; Testa et al., 2001). Given the crucial role that prosody plays in everyday communication, it is important to delineate more precisely the nature of these impairments.

Considering the range of information conveyed by prosody, it is also important to determine whether individuals with AD exhibit 
impairments in the processing of linguistic aspects of prosody. The question of whether impairments in grammatical-prosodic processing are seen in AD has been little-explored to date, although Perez Trullen \& Mondrego Pardo (1996) report impairments in the comprehension of "intrinsic prosody" (distinguishing between statements and questions) in 8 of $25 \mathrm{AD}$ individuals (32\% of their sample). Likewise, in their investigation of comprehension of emotional prosody, Koff, Zaitchik, Montepare, and Albert (1999) included a control task in which AD participants heard 12 low-pass filtered sentences which were either statements, questions, or commands ( $n=4$ of each); AD participants performed significantly worse than control subjects on this task.

While some studies have examined a specific aspect of prosodic processing in $\mathrm{AD}$, there are virtually no studies that have explored a broad range of prosodic domains to evaluate which aspects of prosodic comprehension are specifically impacted by the disease. The goal of the current study is thus to characterize prosodic comprehension skills in AD by means of detailed domain-specific prosodic tests. In addition to testing emotion processing ability, we were also interested in examining three aspects of linguistic prosody comprehension, namely lexical stress perception, detection of grammatical modality, and sensitivity to prosodic boundaries signaling phrase structure. As an initial step, we wished to assess the feasibility of administering such tests in a small sample of patients and healthy elderly controls to identify those prosodic domains in which there is suggestive evidence of impairment. Finally, we were also interested in examining possible correlations of scores obtained from standardized memory (Wechsler memory scale III, Wechsler, 1997) and cognitive (Mini-Mental State Examination, Folstein, Folstein \& McHugh, 1975; MMSE) scales with the results obtained from each of the prosodic tests, in order to ascertain the relationship between memory and global cognitive performance and prosodic comprehension ability among the AD participants assessed.

The range of tasks included in this study allows us to assess whether prosody comprehension impairments are specific to a particular aspect of prosodic processing (e.g., at a lexical level or in affective processing), whether a low-level perceptual impairment underlies the prosodic processing deficits, and/or whether higher-level cognitive skills are related to any of the prosodic impairments found. This approach may elucidate the underlying deficits that lead to prosody comprehension impairments in AD.

\section{Method}

\section{Participants}

Ten participants with mild AD (defined as individuals with MMSE scores $\geq 19$; see, e.g., Aisen et al., 2006; Alzheimer's disease Neuroimaging Initiative, http://www.loni.ucla.edu/ADNI/) were recruited for the study. Diagnosis of probable $\mathrm{AD}$ was established by a neurologist or neuropsychologist according to diagnostic criteria for dementia (Diagnostic and Statistical Manual of Mental Disorders, Fourth Edition; American Psychiatric Association, 1994) and all were diagnosed as having probable AD according to standard clinical criteria (McKhann et al., 1984). Standard blood work and neuroimaging (CT or MRI) were also carried out, and the diagnosis was supported by abnormal performance on neuropsychological testing. Global dementia severity was ascertained on the basis of the MMSE. Ten healthy elderly control participants also took part in the study. Participants' demographic characteristics and psychometric test scores are provided in Table 1. Participant groups did not differ significantly in terms of age, $t(18)=1.58, p=.13$, or education, $t(18)=-0.98$, $p=.34$, although normally aging participants were slightly younger $(d=0.71)$ and more educated $(d=0.44)$ than $\mathrm{AD}$ participants. The two participant groups differed significantly in MMSE scores, $t(18)=-3.55, p<.01$, Wechsler memory subscale-immediate recall scores, $t(18)=-6.61, p<.01$, and delayed recall scores, $t(18)=-10.63, p<.01$. However, the groups did not differ significantly in their performance on letternumber sequencing, $t(18)=-0.90, p=.381$.

All AD participants were recruited from the Memory Clinic of the Jewish General Hospital in Montreal, a tertiary referral center. Control participants were recruited through newspaper advertisements or from the Herzl Family Clinic at the Jewish General Hospital, and underwent a complete neuropsychological battery to exclude dementia and mild cognitive impairment. Procedures were thoroughly explained to all participants, who signed an Informed Consent form approved by the Jewish General Hospital Ethics Review Board. Given that AD patients were at the mild stage, they were deemed capable of providing informed consent. Participants were remunerated for their participation. To confirm that participants' hearing thresholds were adequate to comprehend the stim-

Table 1

Participant Characteristics

\begin{tabular}{|c|c|c|c|c|}
\hline & $\begin{array}{c}\text { Control participants- } \\
\text { mean }(S D)\end{array}$ & $\begin{array}{l}\text { Control participants- } \\
\text { range }\end{array}$ & $\begin{array}{l}\text { AD participants- } \\
\text { mean }(S D)\end{array}$ & $\begin{array}{l}\text { AD participants- } \\
\text { range }\end{array}$ \\
\hline Age & $79.1(6.8)$ & 69 to 88 & $83.1(4.3)$ & 78 to 92 \\
\hline Sex & $6 \mathrm{~F} / 4 \mathrm{M}$ & & $4 \mathrm{~F} / 6 \mathrm{M}$ & \\
\hline Education & $13.1(3.3)$ & 8 to 18 & $11.4(4.4)$ & 7 to 22 \\
\hline Native language $^{\mathrm{a}}$ & 10 English & & $\begin{array}{c}8 \text { English, } 1 \\
\text { Polish, } 1\end{array}$ & \\
\hline & & & English/Arabic & \\
\hline Handedness & $9 \mathrm{right} / 1 \mathrm{left}$ & & 9 right/1 left & \\
\hline MMSE (/30) & $28.7(2.2)$ & 23 to 30 & $24.9(2.6)$ & 21 to 28 \\
\hline Letter-number sequencing (\%ile) & $56.6(26.8)$ & 2 to 91 & $45.7(27.5)$ & 5 to 84 \\
\hline Wechsler memory subscale-immediate recall (\%ile) & $83.7(19.0)$ & 50 to 99 & $24.5(21.0)$ & 0.4 to 63 \\
\hline Wechsler memory subscale-delayed recall (\%ile) & $81.4(20.6)$ & 50 to 99 & $7.6(7.7)$ & 1 to 25 \\
\hline
\end{tabular}

Note. $\quad \mathrm{AD}=$ Alzheimer's disease.

a "Native speaker" defined as learned before the age of 5, speaks without an accent, uses on a daily basis. 
uli, they were required to repeat a series of five sentences played through computer speakers. All participants successfully completed this pretest.

\section{Procedure}

Data were collected in participants' homes, over a period of approximately two hours; if participants reported fatigue, testing took place over two sessions, approximately one week apart. This procedure was necessary for one control and eight AD participants. Each control participant completed the tasks in a different semirandomized order; the order of presentation was matched for $\mathrm{AD}$ and control participants. Stimuli were digitally recorded by a native speaker of English and presented over computer speakers, with the volume set to a comfortable level. Stimuli were replayed as many times as necessary, at the participant's request, although repetition was rarely needed.

To validate previous research indicating impairments in affectiveprosodic comprehension in $\mathrm{AD}$, we included one task examining emotional prosody (Task 1 ) in which we manipulated the availability of supportive linguistic content through the use of nonsense utterances and low-pass filtering of the stimuli. Linguistic prosody comprehension was assessed with several tasks examining lexical stress perception (Task 2), detection of grammatical modality of sentences (Tasks 3, 4, and 5), and sensitivity to prosodic boundaries signaling phrase structure (Tasks 6 and 7). In all tasks, participants heard stimuli in the same pseudorandomized order.

\section{Task 1: Sentence Prosody: Affective Prosody Processing}

In this task, taken from Pell and Baum (1997), participants identified intonation meaning from sentences varying in affective modality and the availability of semantic information (angry, happy, or sad; $n=8$ of each modality in each language condition). English sentences contained sufficient semantic information to determine the modality. These were then low-pass filtered at $500 \mathrm{~Hz}$ to remove most intelligible linguistic information, while conserving intonational variations across the utterances. A third set of sentences used nonsense words; modality was indicated via prosodic information. Participants heard each sentence and indicated orally the affective modality.

\section{Task 2: Lexical-Stress Perception}

Lexical-stress perception was assessed using the Greenhouse test (Pell, 1996), in which participants must differentiate between compound words (e.g., 'greenhouse) and adjective-noun pairs (green 'house; $n=12$ per condition). For each stimulus, participants viewed three pictures simultaneously, one of the referent of the compound, one of the referent of the noun phrase, and one unrelated foil, and selected the picture that matched the stimulus.

\section{Task 3: Sentence Prosody: Sentence Modality I}

Participants determined the grammatical modality (statement or question) of 12 pairs of sentences with either declarative intonation (e.g., "He speaks French.") or interrogative intonation (e.g., "He speaks French?"). The sentence pairs differed only with respect to intonation and were presented in pseudo-randomized order.

\section{Task 4: Sentence Prosody: Sentence Modality II}

This task, taken from Pell and Baum (1997), was identical to Task 1 above, except that participants were required to identify linguistic modality (imperative, declarative, or interrogative) rather than affective tone. Syntactic information provided an additional cue in the English condition, as canonical syntactic structures for statements, questions, and commands were used.

\section{Task 5: Perceptual Categorization of Phrases Varying Along the Statement-Question Continuum}

To assess the establishment of prosodic category boundaries, the $\mathrm{F}_{0}$ of the final word of a natural declarative statement was linearly increased to a level equivalent to that of its question counterpart (see Figure 1). Eight intermediate sentences were created by increasing the final word's $\mathrm{F}_{0}$ in $11 \%$ steps; a similar series was created by transforming the natural question counterpart into a statement. Participants heard each of the resultant 20 sentences twice and categorized each as statement or question.
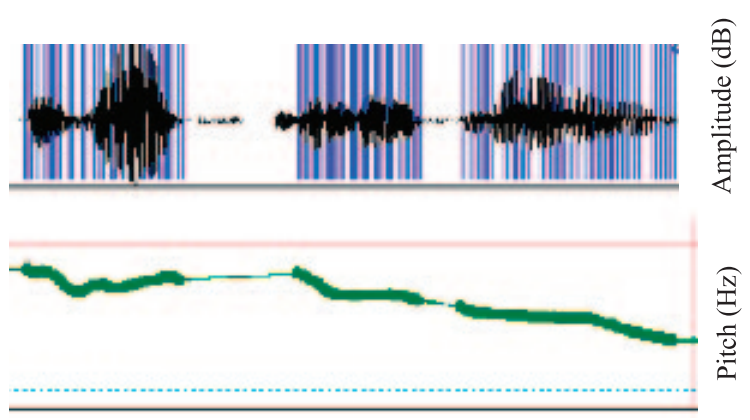

Duration

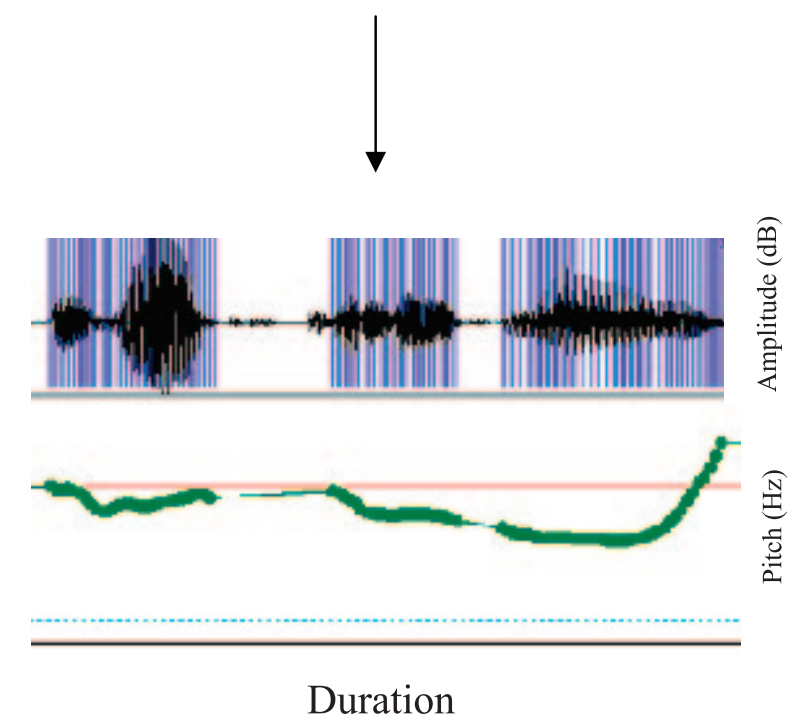

Figure 1. Digital transformation of the statement "He wants to leave now" to the question "He wants to leave now?" 


\section{Task 6: Temporal Cues Marking Phrasal Boundaries}

The phrase "pink and black and green" was manipulated by inserting a prosodic boundary after "pink" or "black" and by varying the pause durations (ranging from 0-160 ms, in five $40 \mathrm{~ms}$ steps) or the preboundary word durations (pink series: 286-446 ms; black series: $284-444 \mathrm{~ms}$, each in five $40 \mathrm{~ms}$ steps; Aasland \& Baum, 2003; Baum, Pell, Leonard, \& Gordon, 1997). Thus, 50 stimuli were created in total: 25 manipulating the prosodic boundary after "pink" (5 preboundary word durations $\times 5$ pause durations) and 25 in which the prosodic boundary after "black" was manipulated. Participants listened to each stimulus and indicated whether they had heard [[pink and black] and green] or [pink and [black and green]], either verbally or using colored squares.

\section{Task 7: Temporal Cues Marking Phrasal Boundaries}

Stimuli comprised 14 pairs of sentences, differing only in the presence of a syntactic/prosodic boundary that changed the phrase structure of the sentence. Stimuli could be interpreted as integrated or nonintegrated. For example, the noun phrase, "my friend", in the sentence, "Paul my friend is handsome," could be the subject of the verb "is" ("Paul, my friend is handsome"; integrated) or an appositive if a prosodic boundary is placed after "my friend" ("Paul, my friend, is handsome"; unintegrated). "Greg", in "My boss told me to call Greg," could be the object of "call" (integrated) or as a tag ("My boss told me to call, Greg"; unintegrated). Seven sentences of each type (tag, appositive) were included in integrated and unintegrated form. After hearing each sentence, participants answered comprehension questions to verify how the sentence was parsed. Sentence pairs were divided into two lists, heard consecutively, such that the two versions of a sentence were never heard in the same half of the experiment. ${ }^{1}$

\section{Results}

\section{Task 1: Sentence Prosody: Affective Prosody Processing}

Figure 2 shows mean group accuracy ratings for each condition. A stimulus type (English, nonsense, low-pass filtered) x emotion (angry,

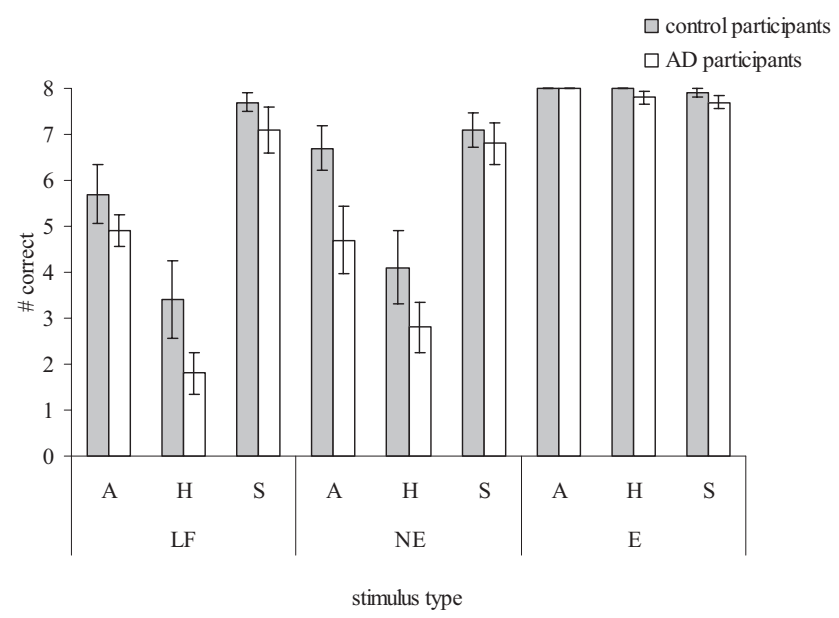

Figure 2. Affective prosody processing. LF: low-pass filtered. NE: nonsense word condition. E: English. A: Angry. H: Happy. S: Sad. Number correct (out of 8) is indicated on the $y$-axis. Error bars represent standard error of the mean.

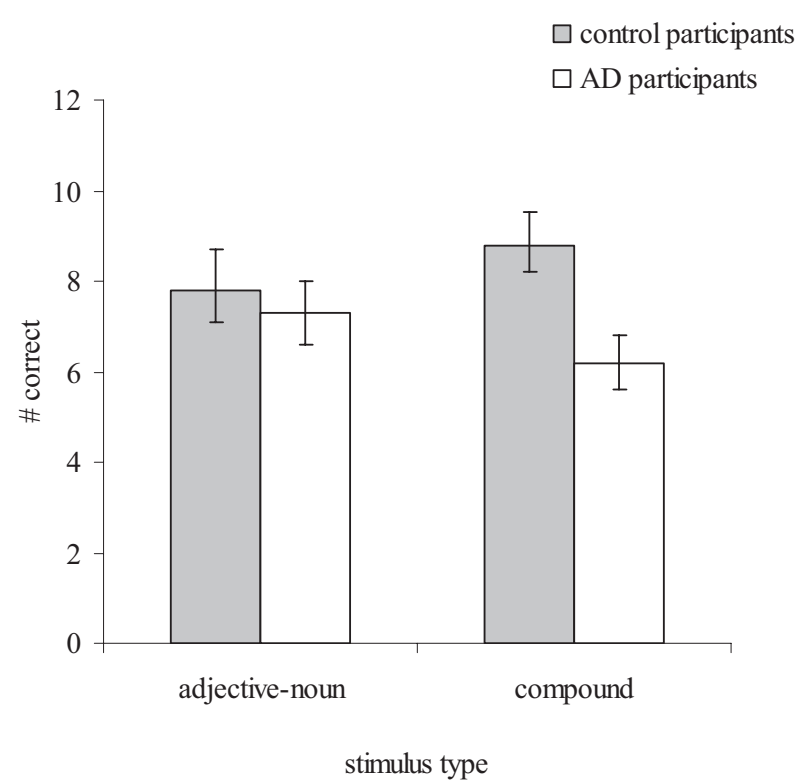

Figure 3. Lexical stress perception. Number correct (out of 12) is indicated on the $y$-axis. Error bars represent standard error of the mean.

happy, sad) x group ANOVA revealed higher accuracy for control than for $\mathrm{AD}$ participants $(F(1,18)=16.53, p<.001$, partial Eta squared $=0.48)$, and for English than for low-pass filtered or nonsense sentences $(F(2,36)=108.47, p<.001$, partial Eta squared $=0.86)$. Sad sentences were better categorized overall than angry sentences, and angry better than happy sentences $(F(2$, $36)=31.17, p<.001$, partial Eta squared $=0.63$ ). In this and other tasks, all post hoc comparisons were conducted using LSD post hoc tests. An interaction between stimulus type and group $(F(2$, $36)=3.65, p<.04$, partial Eta squared $=0.17$ ) was due to lower accuracy rates for AD than control participants on low-pass filtered and nonsense sentences ( $p<.01$ in both cases); a borderline effect was found in English sentences $(p<.054$; means $(S D$ s): AD participants, LF sentences, 4.6 (2.6), nonsense sentences, 4.8 (2.6), English sentences, 7.8 (0.38); control participants, LF sentences, 5.6 (2.6), nonsense sentences, 6.0 (2.2), English sentences, 8.0 (0.18)). Across groups, fewer errors were committed on sad than angry sentences, and on angry than happy sentences in low-pass filtered and nonsense conditions (stimulus type $\mathrm{x}$ emotion $(F(4,72)=16.57, p<.001$, partial Eta squared $=0.48$; means $(S D s)$ : LF sentences, sad, 7.4 (1.2), angry, 5.3 (1.7), happy, 2.6 (2.2); nonsense sentences, sad, 7.0 (1.3), angry, 5.7 (2.2), happy, 3.5 (2.2)). In English stimuli, more errors were committed on sad than angry sentences $(p<.042)$; no other differences between conditions emerged (means ( $S D \mathrm{~s})$ : sad, 7.8 (0.41), angry, $8.0(0)$, happy, $7.9(0.4))$.

\section{Task 2: Lexical-Stress Perception}

Group means for each stimulus type are shown in Figure 3. A group x stimulus type ANOVA revealed no significant main ef-

\footnotetext{
${ }^{1}$ A further 6 ambiguous sentences were also presented, but are not discussed here due to very poor performance by control subjects. The ambiguity in these sentences rested in determining the addressee of the sentence; for example, "Say the sentence "you are handsome, Henry", vs. "Say the sentence, 'you are handsome', Henry."
} 
fects or interactions, although a borderline effect of group $(F(1$, $18)=3.65, p>.07$, partial Eta squared $=0.17$ ) was found. Older adults performed well below ceiling, with an average accuracy rate of only $69 \%$.

\section{Task 3: Sentence Prosody: Sentence Modality I}

Group mean accuracy for each modality is shown in Figure 4, panel 1. AD participants committed more errors than control participants $(F(1,18)=22.11, p<.001$, partial Eta squared $=0.55)$, and accuracy was lower for questions than statements $(F(1,18)=35.89, p<.001$, partial Eta squared $=0.67)$. AD participants committed more errors on questions than control participants (modality $\mathrm{x}$ group: $F(1$, $18)=24.68, p<.001$, partial Eta squared $=0.58$; means $(S D s)$ : control participants, statements: 11.8 (0.4) questions: 11.1 (1.7); AD participants, statements: 11.6 (1.0) questions: 4.1 (4.1)).

\section{Task 4: Sentence Prosody: Sentence Modality II}

Figure 4, panel 2 shows mean group accuracy for each condition. A group $\mathrm{x}$ stimulus type $\mathrm{x}$ modality ANOVA demonstrated that accuracy was higher for control than AD participants $(F(1$, $18)=14.06, p<.001$, partial Eta squared $=0.44)$, and for English than low-pass filtered or nonsense sentences $(F(2$, 36) $=79.86, p<.001$, partial Eta squared $=0.82$ ). Fewer errors were committed on questions than on statements, and on statements than on commands $(F(2,36)=76.62, p<.001$, partial Eta squared $=0.81)$. Interactions were seen between modality and group $(F(2,36)=6.39, p<.004$, partial Eta squared $=0.26)$, modality and stimulus type $(F(4,72)=24.49, p<.001$, partial Eta squared $=0.58)$, and modality, stimulus type, and group $(F(4,72)=4.24, p<.004$, partial Eta squared $=0.19) . \mathrm{AD}$ participants committed more errors than control participants on commands across all three stimulus types (Mean $(S D)$ : English stimuli: AD: 6.6 (1.6), control: 7.9 (0.3); nonsense stimuli: AD: 0.7 (1.1), control: 3.9 (1.8); low-pass filtered stimuli: AD: 1.3 (1.8). control: $3.5(1.8)$ ), on questions in the nonsense condition (AD: 6.8 (1.3); control: $7.9(0.3)$ ), and on statements in the English language condition (AD: 6.8 (1.3); control: $7.8(0.4)$ ). Overall, $87 \%$ of errors on questions and $83 \%$ of errors on commands comprised misclassifications as statements.
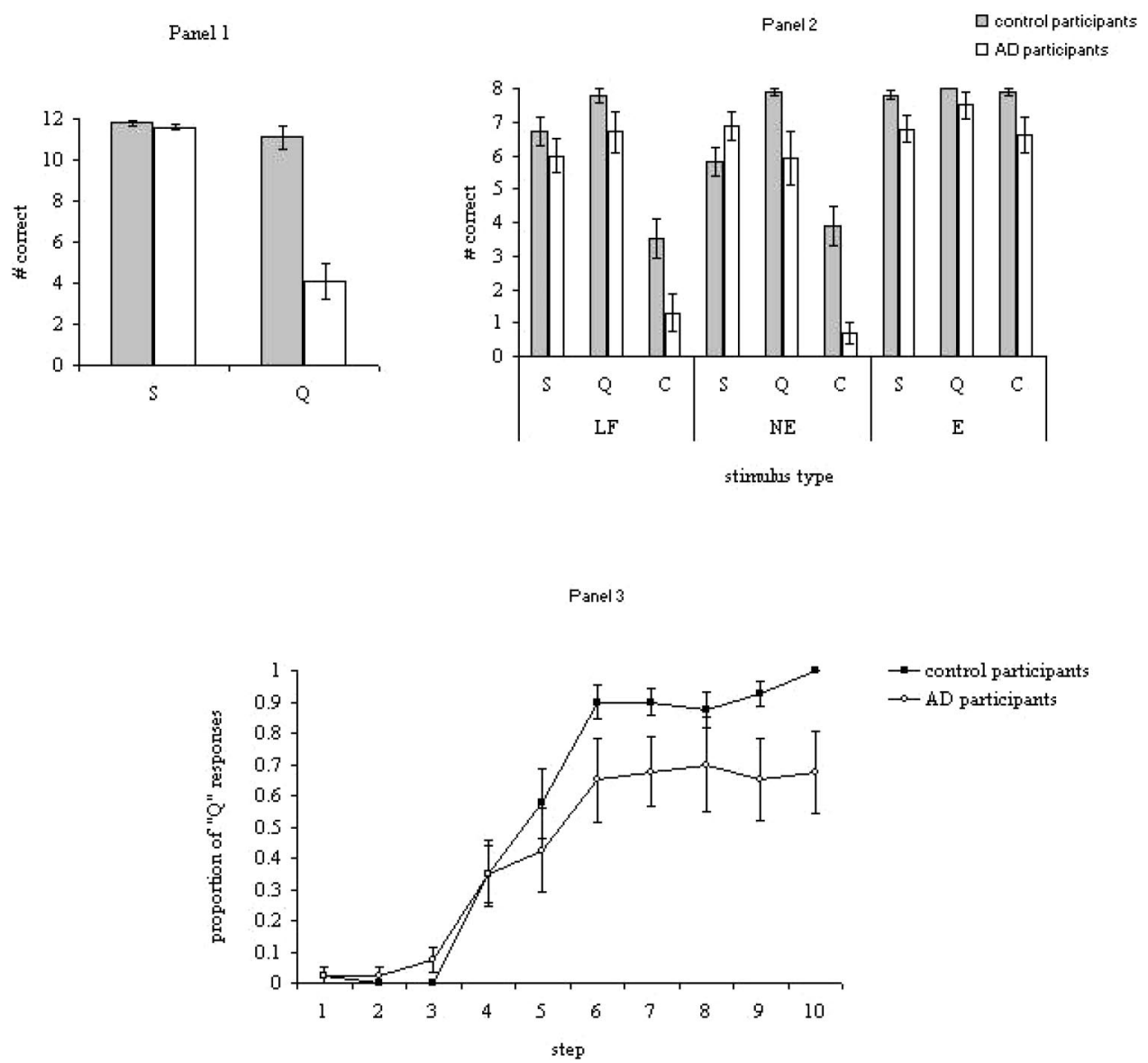

Figure 4. 1: Sentence modality categorization I. Sentence type is indicated on the $x$-axis. S: statement. Q: question. Number correct (out of 12) is indicated on the $y$-axis. 2: Sentence modality categorization II. LF: low-pass filtered. NE: nonsense word condition. E: English. S: statement. Q: question. C: command. Number correct (out of 8) is indicated on the $y$-axis. Error bars represent standard error of the mean. 3: Categorical perception. Percentage of "question" responses is indicated on the $y$-axis. Step is indicated on the $x$-axis (with 1 being most statement-like and 10 being most question-like). 
Task 5: Perceptual Categorization of Phrases Varying Along the Statement-Question Continuum

Figure 4, panel 3 shows group mean percent "question" responses for each step on the continuum. Each participant's category crossover boundary was determined by calculating the intercept and slope of the curve and then applying the following formula: boundary $=(0.5$-intercept $) /$ slope (e.g., Miller \& Dexter, 1988). The boundary indicates the point at which participants respond at about chance level. No crossover boundary could be calculated for three AD participants, who never consistently responded "question". The average boundaries occurred at step 4.54 for the remaining $\mathrm{AD}$ participants and at 4.96 for control participants. No significant group differences emerged, $t(18)=0.714$, $p=.49$.

Because boundary values largely reflect responses in the ambiguous region of the continuum, and group performance may also differ at the continuum endpoints, an analysis was conducted on overall percent question responses across the continuum (see Pitt \& Samuel, 1993, for similar analyses). A group x step ANOVA revealed a main effect of step, $F(9,162)=50.33, p<.001$. AD and control participants differed significantly at step 10 (step $\mathrm{x}$ group $(F(9,162)=2.74, p<.005$, partial Eta squared $=0.13)$, and a borderline effect was seen at step $9(p<.058$; mean $(S D)$ : step 10, control participants, 100 (0); AD participants, 67.5 (40.9); step 9, mean $(S D)$ : control participants, 92.5 (12.1); AD participants, $65.0(41.2))$.

\section{Task 6: Temporal Cues Marking Phrasal Boundaries}

Group mean number of p_bg (i.e., black and green grouped together) responses was calculated as a function of keyword duration (Figure 5, Panel 1) and pause duration (Figure 5, Panel 2). Thus, the data in Panel 1 collapse the different pause durations to give an average number of p_bg responses for each keyword duration, and Panel 2 collapses the keyword durations to give an average number of p_bg responses for each pause duration.

Two separate ANOVAs were conducted to determine the effect of keyword duration and pause duration. In both, more p_bg responses were seen in the pink than in the black series (keyword duration analysis: $F(1,18)=140.73, p<.001$, partial Eta squared $=0.89$; pause duration analysis: $F(1,18)=323.34, p<$ .001 , partial Eta squared $=0.95$ ). No significant group effects or interactions emerged in either analysis.

\section{Task 7: Temporal Cues Marking Phrasal Boundaries II}

Figure 6 shows group means for each condition. A group $\mathrm{x}$ sentence type $\mathrm{x}$ integration ANOVA yielded a main effect of sentence type $(F(1,18)=66.61 ; p<.001$, partial Eta squared $=0.79$ ). Both groups performed better on tag constructions than on appositives. No effect of group or integration, nor any interaction, was found.

\section{Correlations With Cognitive Function}

In order to assess the influence of overall cognitive function on AD participants' performance on prosodic tasks, Pearson correlation coefficients were calculated between scores on each task and scores on standardized tests of memory and cognition (MMSE, letter-number sequencing, immediate and delayed recall). No significant correlations were seen between any of these measures $(p>.1$ in all cases).

\section{Discussion}

The goal of the present pilot study was to examine comprehension of different subtypes of prosody in AD. Overall, processing of information relating to affective tone and grammatical modality was found to be impaired, while information relating to syntactic structure and lexical stress information appeared relatively spared.

Impairments in comprehension of affective-prosodic information in $\mathrm{AD}$ emerged primarily when affective modality was signaled solely by prosody (i.e., in low-pass filtered and nonsense stimuli). This finding suggests that individuals with AD can still take advantage of semantic/pragmatic information in the speech signal to compensate for their affective-prosodic processing impairments, consistent with research indicating that $\mathrm{AD}$ individuals
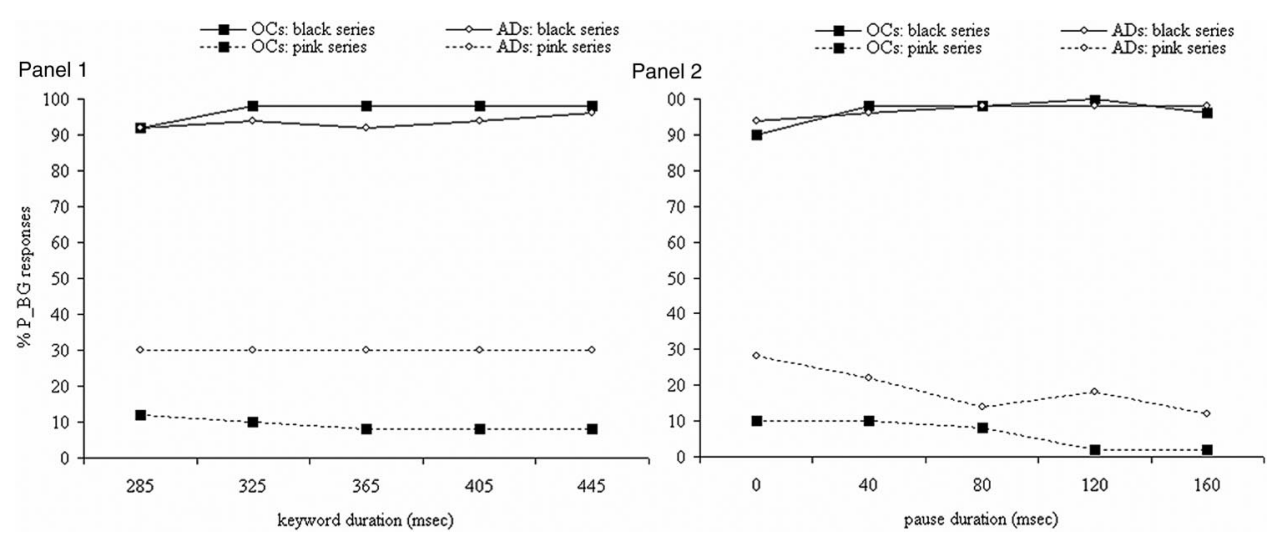

Figure 5. Perceptual prosody processing II: effects of pause (panel 1) and keyword (panel 2) duration. Percentage of "p_bg" responses (i.e., grouping black and green together) is shown on the $y$-axis. P-series: duration of the keyword "pink"/duration of pause following "pink". B-series: duration of the keyword "black"/ duration of pause following "black". Average keyword durations across the pink and black series are marked on the $x$-axis; the duration of the keyword "black" was $1 \mathrm{~ms}$ shorter and the duration of the keyword "pink" was 1 $\mathrm{ms}$ longer than the values given at each step. 


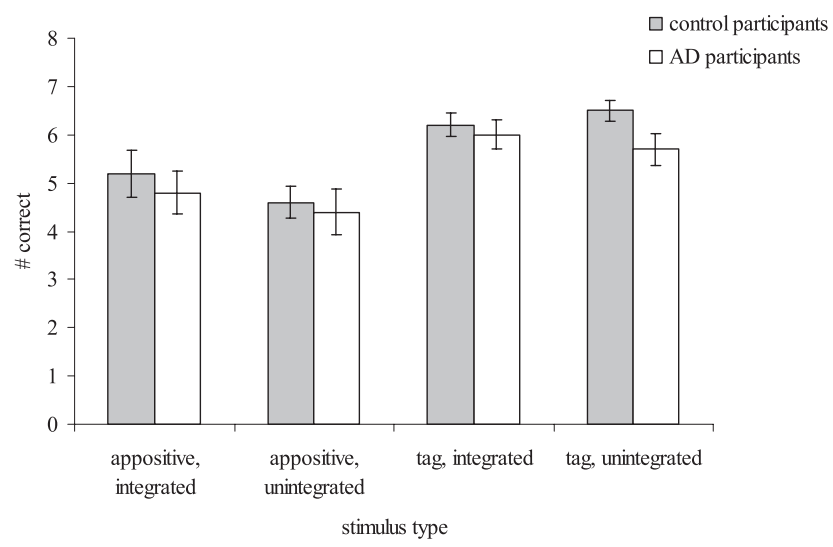

Figure 6. Temporal cues marking phrasal boundaries. Number correct (out of 7) is indicated on the $y$-axis. Error bars represent standard error of the mean.

tend to benefit disproportionately from sentence context in lexical processing (e.g., Schwartz, Federmeier, Van Petten, Salmon, \& Kutas, 2003).

Interestingly, both participant groups manifested error rates over $50 \%$ in distinguishing happy from both angry and sad sentences in the low-pass filtered and nonsense conditions; no consistent pattern could be seen in terms of the erroneous responses. These apparent impairments in affective-prosodic processing in healthy older adults are consistent with previous literature (Brosgole \& Weisman, 1995; Cohen \& Brosgole, 1988; Kiss \& Ennis, 2001; Ross, Orbelo, Testa, \& Beatty, 2000). For instance, Pell and Baum (1997) found that their healthy older participants exhibited similar difficulty in the "happy" condition. Although it is not immediately apparent why "happy" stimuli are more difficult to categorize, the dissociation among the emotional prosody categories merits exploration in future research.

With respect to AD participants' comprehension of linguistic prosody, the primary impairments emerged in classification of sentences' grammatical modality, particularly when semantic/ pragmatic information was unavailable. This performance pattern suggests that patients were unable, in the majority of cases, to utilize prosodic information to determine grammatical modality; we postulate that they relied upon available — sometimes misleading - syntactic information to categorize the sentences as statements. Findings from the perceptual categorization task are consistent with these claims: while AD and control participants did not differ in their use of the sentence-final word's $F_{0}$ to distinguish statements from questions, $\mathrm{AD}$ participants produced significantly fewer "question" responses at the question end of the continuum, and $3 \mathrm{AD}$ participants did not shift from statement to question at any point in the continuum. This finding suggests that at least a subset of AD patients are impaired in distinguishing grammatical modality using only prosodic information; alternatively, these individuals may exhibit a "statement" response bias. These results are consistent with previous research (Perez Trullen \& Mondrego Pardo, 1996). The "statement" bias may be due to a reliance on the typical or default sentence structure associated with declarative utterances.

That AD individuals exhibited impairments in comprehension of emotional prosody and sentence modality, but largely intact performance in the remaining tasks, suggests that prosody comprehension impairments in $\mathrm{AD}$ cannot be solely attributed to grammatical, emotional, perceptual, or cognitive deficits. Rather, it appears that prosody comprehension may be impaired independently of other cognitive or perceptual deficits in this population. One possibility is that $\mathrm{AD}$ patients experience difficulties in processing prosodic information signaled across an entire sentence. Note that $\mathrm{AD}$ and control groups did not differ on a working memory measure, suggesting that this impairment is unlikely to be due to working memory performance. This hypothesis should be explored further with larger and more varied participant groups.

No correlations were seen between prosody comprehension and cognitive function, consistent with the hypothesis that prosody comprehension impairments occur early in the disease course and remain stable as cognitive function declines. It should be noted, however, that all patients were in the mild stage of the disease, that MMSE scores were distributed within a relatively narrow range, and that limited data points are available; thus, these findings must be interpreted with caution. Additionally, it is possible that AD individuals had difficulty understanding the tasks and thus exhibited lower performance than control participants. However, AD participants' intact performance in certain tasks and conditions, as well as the fact that their cognition was only mildly impaired, imply that the present findings are not solely attributable to a failure to comprehend the tasks, but rather indicate a genuine prosodic impairment.

A second issue that arises in interpreting the current findings is sample size. Since the present study was a feasibility study aiming to assess the sensitivity of different tasks to decline in AD, relatively few participants were included. Nonetheless, effect sizes were reasonably high in tasks assessing affective prosody comprehension and detection of grammatical modality. While control participants were slightly younger and more educated than $\mathrm{AD}$ participants, these effects did not reach significance and were not likely major influences on the results that emerged. The present findings suggest that the tasks used here are suitable for assessment of decline in prosody comprehension in $\mathrm{AD}$, paving the way for a larger-scale study focused on the specific tasks most sensitive to decline.

In conclusion, the present findings suggest that impairments in both grammatical and emotional prosody comprehension are seen in AD. These impairments appear to be most severe when prosodic information is signaled across a sentence, and are present even very early in the disease course. Given the crucial role that prosody plays in successful communication, a clearer understanding of the nature of prosodic processing impairments in $\mathrm{AD}$ will have important implications in terms of intervention, indicating potential strategies to improve communication with these individuals. For example, the finding that $\mathrm{AD}$ patients are able to use semantic information to compensate for prosody comprehension impairments points to strategies for more efficient communication through provision of supporting contextual information. Similarly, their relatively intact syntactic comprehension abilities provide an important tool to support the use of grammatical prosody.

Given that comprehension of emotional prosody has been shown to correlate closely with disease progression (Testa et al., 2001), future research should examine comprehension of different prosody subtypes at different stages in the disease. The current study included AD patients at a very early stage in the disease (average MMSE = 24.9; range 21-28). Given Testa et al.'s (2001) 
findings, it seems likely that the impairments reported here would become more severe as the disease progresses. Whether impairments in comprehension across different prosodic functions would appear later in the evolution of the disease remains an open question. Likewise, the heterogeneous nature of $\mathrm{AD}$ is wellknown; as such, it is of interest to pursue the notion of subgroups of $\mathrm{AD}$ individuals with differing levels of prosodic impairment. Finally, the present findings should be extended to prosody production capacity in $\mathrm{AD}$, about which little is currently known.

\section{References}

Aasland, W. \& Baum, S. R. Temporal parameters as cues to phrasal boundaries: A comparison of processing by left- and right-hemisphere brain damaged individuals. Brain and Language, 87, 385-399.

Aisen, P. S., Saumier, D., Briand, R., Laurin, J., Gervais, F., Tremblay, P., et al. (2006). Phase II study targeting amyloid-beta with 3 APS in mild-to-moderate Alzheimer disease. Neurology, 67, 1757-1763.

Albert, M., Cohen, C., \& Koff, E. (1991). Perception of affect in patients with dementia of the Alzheimer type. Archives of Neurology, 48, 791795 .

Allender, J., \& Kaszniak, A. (1989). Processing of emotional cues in patients with dementia of the Alzheimer's type. International Journal of Neuroscience, 46, 147-155.

American Psychiatric Association. (1994). Diagnostic and statistical manual of mental disorders (4th ed.). Washington, DC: Author.

Baum, S. R., Pell, M., Leonard, C., \& Gordon, J. (1997). The ability of right- and left-hemisphere-damaged individuals to produce and interpret prosodic cues marking phrasal boundaries. Language and Speech, 40, 313-330.

Baum, S. R., \& Pell, M. D. (1999). The neural bases of prosody: Insights from lesion studies and neuroimaging. Aphasiology, 13, 581-608.

Brosgole, L., \& Weisman, J. (1995). Mood recognition across the ages. International Journal of Neuroscience, 82, 169-189.

Cadieux, N., \& Greve, K. (1997). Emotion processing in Alzheimer's disease. Journal of the International Neuropsychological Society, 3, 411-419.

Caramelli, P., Mansur, L. L., \& Nitrini, R. (1998). Language and communication disorders in dementia of the Alzheimer type. In B. Stemmer \& H. A. Whitaker (Eds.), Handbook of Neurolinguistics (pp. 463-473). San Diego, CA: Academic Press.

Cohen, E., \& Brosgole, L. (1988). Visual and auditory affect recognition in senile and normal elderly persons. International Journal of Neuroscience, 43, 89-101.

Folstein, M. J., Folstein, S. E., \& McHugh, P. R. (1975). Mini-mental state: A practical method for grading the cognitive state of the patients for the clinician. Journal of Psychiatric Research, 12, 189-198.
Hodges, J. R., \& Patterson, K. (1995). Is semantic memory consistently impaired early in the course of Alzheimer's disease? Neuroanatomical and diagnostic implications. Neuropsychologia, 33, 441-459.

Kertesz, A. (1982). Western Aphasia Battery. New York: Grune \& Stratton.

Kiss, I., \& Ennis, T. (2001). Age-related decline in perception of prosodic affect. Applied Neuropsychology, 8, 251-254.

Koff, E., Zaitchik, D., Montepare, J., \& Albert, M. S. (1999). Emotion processing in the visual and auditory domains by patients with Alzheimer's disease. Journal of the International Neuropsychological Society, 5, 32-40.

McKhann, G., Drachman, D., Folstein, M., Katzman, R., Price, D., \& Stadlan, E. M. (1984). Clinical diagnosis of Alzheimer's disease: Report of the NINCDS-ADRDA work group under the auspices of Health and Human Services Task Force on Alzheimer's Disease. Neurology, 34, 939-944.

Miller, J., \& Dexter, E. (1988). Effects of speaking rate and lexical status on phonetic perception. Journal of Experimental Psychology: Human Perception and Performance, 14, 369-378.

Pell, M. (1996). On the receptive prosodic loss in Parkinson's disease. Cortex, 32, 693-704.

Pell, M. D., \& Baum, S. R. (1997). The ability to perceive and comprehend intonation in linguistic and affective contexts by brain damaged adults. Brain and Language, 57, 80-99.

Perez Trullen, J. M., \& Mondrego Pardo, P. J. (1996). Comparative study of aprosody in Alzheimer's disease and in multi-infarct dementia. Dementia, 7, 59-62.

Pitt, M., \& Samuel, A. (1993). An empirical and meta-analytic evaluation of the phoneme identification task. Journal of Experimental Psychology: Human Perception and Performance, 19, 699-725.

Roberts, V., Ingram, S. M., Lamar, M., \& Green, R. C. (1996). Prosody impairment and associated affective and behavioral disturbances in Alzheimer's disease. Neurology, 47, 1482-1488.

Ross, E., Orbelo, D., Testa, J., \& Beatty, W. (2000). Age-related changes in processing affective prosody. Neurology, 54(Suppl 3), A418-A419.

Schwartz, T. J., Federmeier, K. D., Van Petten, C., Salmon, D. P., \& Kutas, M. (2003). Electrophysiological analysis of context effects in Alzheimer's disease. Neuropsychology, 17, 187-201.

Testa, J. A., W. W. Beatty, A. C. Gleason, D. M. Orbelo, \& E. D. Ross. (2001). Impaired affective prosody in AD: Relationship to aphasic deficits and emotional behaviors. Neurology, 57, 1474-1481.

Wechsler, D. (1997). WMS III: Administration and Scoring Manual. San Antonio, TX: Harcourt.

Received January 15, 2007

Revision received September 5, 2007

Accepted September 10, 2007 